\title{
SEARCH FOR FAR-IR PAH BANDS WITH HERSCHEL: MODELLING AND OBSERVATIONAL APPROACHES
}

\author{
C. Joblin ${ }^{1,2}$, G. Mulas ${ }^{3}$, G. Malloci ${ }^{3}$ and E. Bergin \& the HEXOS \\ consortium $^{4}$
}

\begin{abstract}
Herschel opens the possibility to detect the low-frequency vibrational bands of individual polycyclic aromatic hydrocarbon (PAH) molecules and therefore to progress in our understanding of the nature of these species and the properties of the environments from which they emit. However, unless one individual molecule dominates the PAH family, this detection will not be straightforward and it is necessary to optimise the observational search with an educated guess of the band profiles and intensities. Such educated guess can be obtained from models that include a detailed description of the molecular properties (anharmonicity, rotation...) in the modelling of the cooling cascade of the emitting species. First results are expected soon from the observation of the Orion Bar as part of the HEXOS Herschel key program.
\end{abstract}

\section{Introduction}

Polycyclic aromatic hydrocarbons (PAHs) are commonly believed to be the carriers of the aromatic infrared bands (AIBs) that dominate the mid-IR emission between 3.3 and $15 \mu \mathrm{m}$ in most galactic and extragalactic objects. However the PAH model suffers from the lack of identification of any individual species and different approaches have been pursued over the years to address this question. Diffuse interstellar bands (DIBs), that fall essentially in the visible range (Herbig 1995), are a way to identify individual PAH molecules by their low-lying electronic transitions, which are very characteristic. However, "fishing" for candidates has not been very successful (see Snow \& Destree and Cox and references in these

\footnotetext{
${ }^{1}$ Université de Toulouse, UPS, CESR, 9 Av. colonel Roche, 31028 Toulouse Cedex 4, France

2 CNRS, UMR 5187, 31028 Toulouse, France

3 INAF - Osservatorio Astronomico di Cagliari - Astrochemistry Group, Strada 54, Loc. Poggio dei Pini, 09012 Capoterra (CA), Italy

4 Department of Astronomy, University of Michigan, 500 Church Street, Ann Arbor, MI 48109, USA
} 
articles, elsewhere in this volume). This approach is a lengthy task considering that each species has specific electronic transitions whose measurement for DIB identification requires dedicated set-ups in the laboratory, to mimic the physical conditions of low pressure and temperature in interstellar space. These experiments are very demanding (see for instance Tan \& Salama 2006; Useli-Bacchitta et al. 2010; Pino et al., elsewhere in this volume) and the number of candidates that need to be investigated is very large. For a given number of carbon atoms, $\mathrm{N}_{C}$, there are different isomeric forms as well as a distribution of hydrogenation and ionisation states ( $c f$. Bierbaum et al. and Montillaud et al., elsewhere in this volume).

Rotational spectroscopy is another technique that has been successful over the years to identify circumstellar and interstellar molecules and radicals with the use of radio telescopes. Dipolar PAHs can display a pure rotational emission spectrum in the radio domain but transitions are expected to be weak; PAHs ought to be rotating suprathermally in photodissociation regions (PDRs), yielding a very large rotational partition function (Rouan et al. 1992; Mulas 1998). Combined emissions from different species will lead to forests of weak rotational lines that would merge into a quasi-continuum; PAHs are likely to contribute to the excess microwave emission (Ysard et al. 2010). A better characterization of the nature of the carriers of the anomalous emission would allow to constrain their spatial variation that is required to retrieve the cosmic microwave background in the studies that are currently led with the Planck mission.

Searches for the vibrational emission features in the far-IR range provide a good alternative to visible (DIB) and radio (rotational) identification methods and that is becoming feasible with the Herschel mission. These features involve the whole carbon skeleton of the molecule and are therefore more specific to the exact molecular identity than the ones in the mid-IR, which instead probe functional groups. Experimental data in the far-IR range are still scarce and await for further progress in the coming years (Zhang et al. 2010; Mattioda et al. 2009; Pirali et al. 2006). On the other hand, more and more data are available from quantumchemistry calculations using density functional theory (DFT; cf. Pauzat in this volume) and are collected in databases (Bauschlicher et al. 2010; Malloci et al. 2007). This opens the possibility to model the far-IR emission of PAHs in PDRs.

\section{Model of the (far-)IR emission spectrum of PAHs}

The IR emission from vibrationally excited PAHs can be calculated from fundamental equations ( $c f$. Allamandola et al. 1989). The main step is to properly describe the populations in all levels $v$ of all modes $i$ during the cooling process. Most of the authors in the literature have used the simplifying assumption of thermal distributions, in which the populations of vibrational levels are described by the Boltzmann equation, with the canonical formalism ( $c f$. for instance Désert et al. 1990; Pech et al. 2002; Draine \& Li 2007). Our studies (Mulas 1998; Joblin et al. 2002; Mulas et al. 2006a) are based on the microcanonical formalism in which the energy is the well-defined, conserved physical parameter. This more accurately 
describes the statistical behaviour of a PAH molecule with a given internal energy $U$ but comes at the price of having to explicitly take into account all vibrational modes. A Monte Carlo technique is used to properly solve the master equation and follow the population of states during the cooling of the PAH down to the ground level. Other studies ( $c f$. Ysard et al. 2010) used the exact-statistical method of Draine \& Li (2007). Comparison of results obtained respectively with the microcanonical formalism and thermal approximation can be found in Joblin \& Mulas (2009). Significant differences have been found for the band intensities (a factor of two or more for some of the bands). The emission spectrum of a large sample of PAHs in different charge states (neutrals and cations) and in specific objects (such as the Red Rectangle nebula) has been computed by Mulas et al. (2006a). The Monte-Carlo code takes as an input molecular parameters that are available in the theoretical on-line spectral database of PAHs (Malloci et al. 2007): the positions and intensities of all vibrational modes and the photo-absorption cross-sections up to the vacuum-UV. This allows an accurate calculation of the band intensities but the band profiles have to be assumed ( $c f$. Fig. 1 and Mulas et al. 2006a).

The far-IR bands are emitted mainly at the end of the cooling cascade. Furthermore, at low excitation, it is necessary to consider that at some point the redistribution of energy between vibrational levels (the so-called internal vibrational redistribution, IVR) breaks down; the vibrational states decouple and their quantum numbers cannot be described any more in statistical mechanics terms, but must instead be described explicitly in terms of specific vibrational transitions between specific states. The population of states at decoupling is governed only by statistics and therefore does not depend on the Einstein coefficients associated with the far-IR modes. However the latter govern the relaxation rate and play a key role in the competition with the UV absorption rate. If the UV rate is slow compared to the relaxation then the far-IR cooling can further proceed and very weakly active or even inactive modes can relax in a manner analogous to the emission of forbidden atomic lines in nebulae. In other cases, absorption of another UV photon triggers again mid-IR emission from the hot PAH, and this leads to reduced emission in the far-IR. In particular, the "slowest" far-IR modes can be totally suppressed ( $c f$. case of IRAS $21282+5050$ in Appendix C of Mulas et al. 2006a).

\section{Detailed calculation of the (far-) IR band profiles}

A key aspect in the detection and identification of far-IR bands due to PAHs is related to the exact band profile. During the cooling cascade of the excited $\mathrm{PAH}$, most of the transitions happen between vibrationally excited states. Due to vibrational anharmonicity, the position and width of the emitted bands will depend on the involved states ( $c f$. Oomens and Basire et al. in this volume). Many far-IR bands are due to out-of-plane vibrational modes, for which one can distinguish two main parts in the ro-vibrational band profile: the $\mathrm{Q}$ branch, in which fine structure is expected due to anharmonicity, and the $\mathrm{P}$ and $\mathrm{R}$ branches, that carry the rotational information. The far-IR bands being preferentially emitted near the 


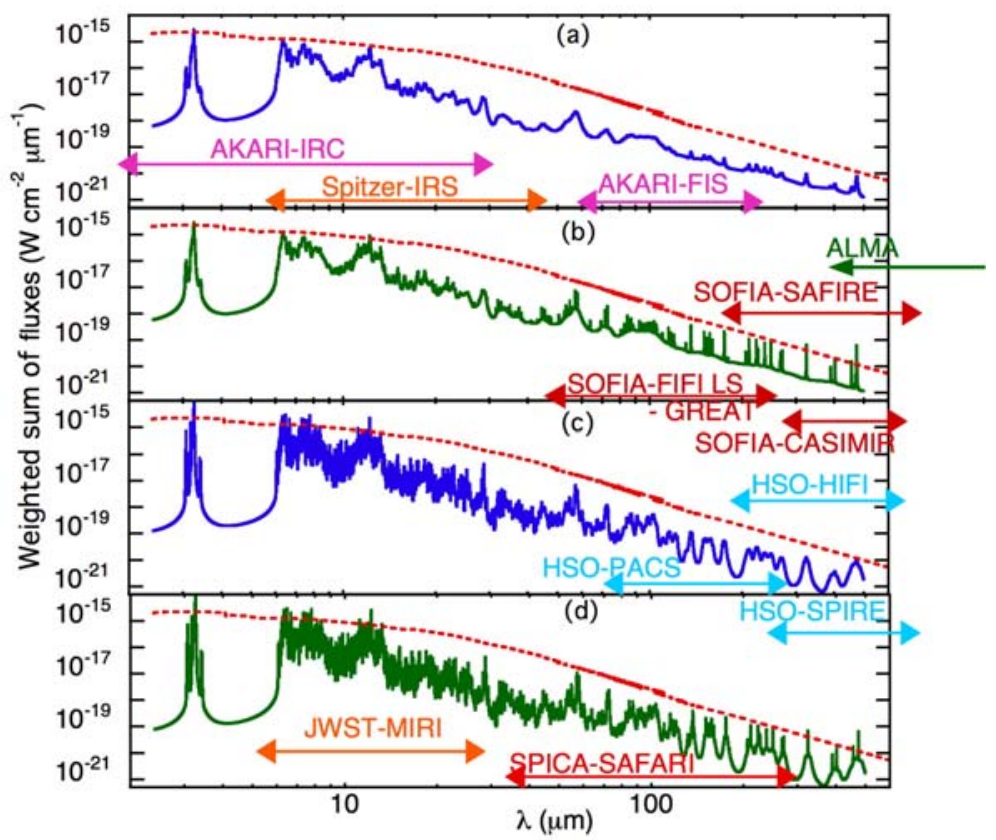

Fig. 1. Calculated infrared emission spectrum from a sample of $40 \mathrm{PAHs}$ ranging in size from $\mathrm{C}_{10} \mathrm{H}_{8}$ to $\mathrm{C}_{48} \mathrm{H}_{20}$ and with neutral or cationic charge, compared with the estimated dust continuum in the Red Rectangle nebula. Different panels correspond to different values for $\sigma_{P R}$, the width of the $\mathrm{P}$ and $\mathrm{R}$ rotational branches, and $\sigma_{Q}$, the width of the central Q branch ( $c f$. Mulas et al. 2006a for more details). The operation ranges of the relevant astronomical facilities are overlaid for reference. Herschel will open the possibility to identify PAHs by their far-IR bands.

end of the cooling cascades, the fine structure of these bands should not be washed out by coupling between states, as it happens for mid-IR bands. In particular, for out-of-plane modes, which have sharp Q branches, this enhances spectral contrast, making their detection possible in spite of the expected strong dust continuum background and spectral crowding (cf. (b) and (d) in Fig. 1).

The modelling of the band profile requires prior knowledge of several molecular parameters: the $\chi$ matrix of anharmonic parameters (including a proper treatment of resonances) and the variations of the rotational constants as a function of the vibrational state. These parameters are not easy to access by experiment or theory; a recent study has been performed on naphthalene, $\mathrm{C}_{10} \mathrm{H}_{8}$ (Pirali et al. 2009), that illustrates the interplay between high-resolution spectroscopy and numerical simulations of the photophysics of PAHs to access these parameters.

In order to compare with astronomical observations, unless one can use directly experimental results of UV-induced IR fluorescence taken under experimental con- 
ditions close to the ones expected in space (but such results are not available yet), some modelling of the emission process is necessary. As previously explained in Section 2, the two commonly used strategies are the simpler, faster, but less accurate thermal approximation, or the more complicated, but more accurate microcanonical approach that we implemented in the form of Monte Carlo simulations ( cf. Mulas et al. 2006a, 2006b). In our Monte Carlo model, the cooling cascades of the PAHs were simulated assuming collisions to be negligible, and furthermore neglecting photodissociation. While photodissociation is expected to be relevant in the ISM, it still occurs on a relatively small fraction of the UV absorption events, resulting in a small correction to the estimated IR emission. Photoionisation is a bit more frequent for neutrals and negative ions, and was taken into account. A large enough number of UV absorption events and relative IR cascades were simulated, to accumulate sufficient statistics on the emission process. The population of rotational modes (in the semirigid rotor approximation), and the statistics of anharmonic shifts were recorded in the simulations, enabling us to produce full rovibrational structures.

\section{PAH far-IR spectroscopy with Herschel}

From an observational point of view, the search for the far-IR bands of PAHs is now becoming possible with the Herschel space observatory (Pilbratt et al. 2010) that has been launched in 2009. Herschel is equipped with three instruments that cover the far-IR and sub-mm ranges from typically 50 to $600 \mu \mathrm{m}$. Detailed presentations of the three instruments, the Photodetector Array Camera and Spectrometer (PACS; Poglitsch et al. 2010), the Spectral and Photometric Imaging REceiver (SPIRE; Griffin et al. 2010) and the Herschel-Heterodyne Instrument for the Far-Infrared (HIFI; de Graauw et al. 2010) can be found elsewhere. The best strategy to detect the PAH far-IR bands is to search for the Q branches associated with the out-of-plane modes. The PACS and SPIRE instruments provide the best compromise between resolving power and sensitivity to evidence these features. Follow-up observations at very high resolution with the heterodyne spectrometer HIFI will allow us to resolve the hot band structure of the Q branches and may be also some structure in the $\mathrm{P}$ and $\mathrm{R}$ branches. These structures, if detected, carry detailed information on the emitting molecule as well as on the local physical conditions that prevail in the emitting environments. To retrieve this information it is necessary to model these features as described in Section 3.

As an example, we present here new calculations of band profiles performed on the large molecule neutral circumovalene $\left(\mathrm{C}_{66} \mathrm{H}_{20}\right)$. These calculations were motivated by the Herschel observations of EXtra-Ordinary Sources (HEXOS; Bergin et al. 2010) key program. In particular, observations are performed on the prototype bright PDR, the Orion Bar, following the strategy explained above. For the molecular parameters, realistic $\chi$ matrices were built based on the knowledge of the smaller molecules $\mathrm{C}_{10} \mathrm{H}_{8}$ and $\mathrm{C}_{14} \mathrm{H}_{10}$ studied by Mulas et al. (2006b). A similar educated guess was performed for the variations of the rotational constants. The radiation field of the Orion Bar was calculated using the Meudon PDR code 


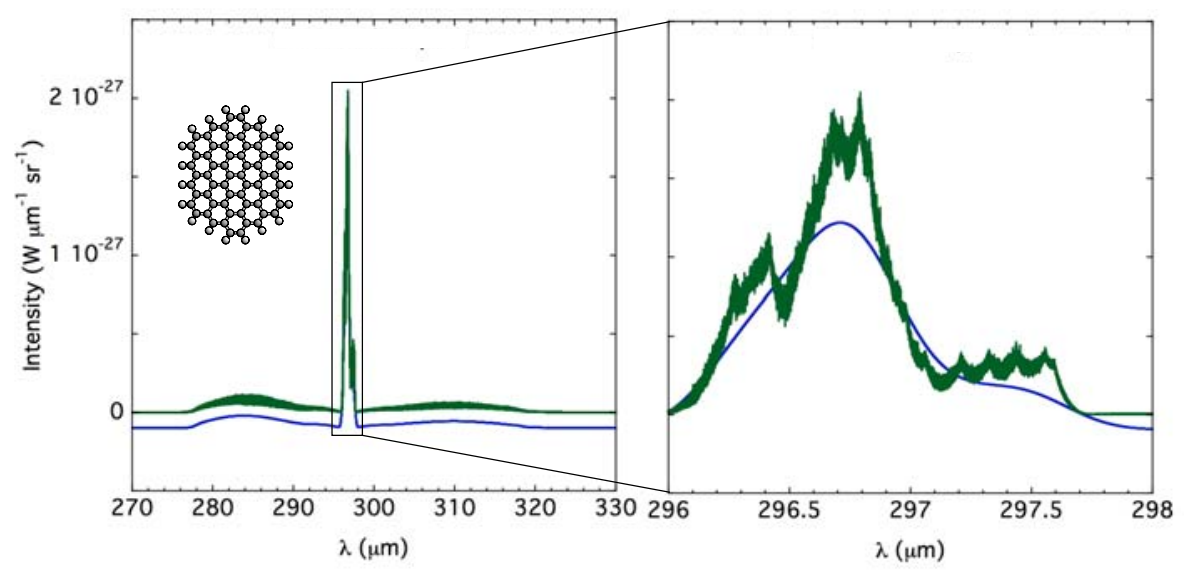

Fig. 2. One example of the calculated structure of the low energy IR-active band of circumovalene $\left(\mathrm{C}_{66} \mathrm{H}_{20}\right)$, computed for one molecule embedded in the Orion Bar radiation field assumed to be $\mathrm{G}_{0}=5 \times 10^{4}$ in Habing units. Full PQR structure (left) and zoom in on the $\mathrm{Q}$ branch (right). Green (upper) spectrum is at full resolution and illustrates the complex structure that could be revealed by the Herschel HIFI heterodyne spectrometer. Blue (lower) spectrum has been convolved at the highest resolution of the Herschel SPIRE instrument.

(Le Petit et al. 2006) with a stellar spectrum from the Kurucz library with a temperature of $40000 \mathrm{~K}$ (Kurucz 1991), a distance of $0.25 \mathrm{pc}$ and a gas density of $\mathrm{n}_{H}=5 \times 10^{4} \mathrm{~cm}^{-3}$. The calculated radiation field at the cloud surface has an integrated UV intensity of $\mathrm{G}_{0}=5 \times 10^{4}$ in Habing units (Habing 1968). The cooling cascades of $\mathrm{C}_{66} \mathrm{H}_{20}$ in the Orion Bar field were then calculated and the band profiles obtained as described in Section 3. The calculated profiles were convolved at the spectral resolution of the instruments onboard Herschel (cf. Fig. 2).

\section{Conclusions and perspectives}

The search for the far-IR bands of PAHs is expected to be a difficult subject since the bands are predicted to be weak and our ability to observe them will depend on how diverse is the mixture of interstellar PAHs. Herschel offers the possibility to investigate this question thanks to the characteristics and the spectral range covered by its instruments. SOFIA and ALMA that are now entering the scene could also bring new insights into this topics. The next far-IR/sub-mm space mission will then be SPICA (2018). But none of these new missions will allow to cover the full Herschel spectral range and we should therefore try hard to search for these PAH bands with Herschel. As we have illustrated, a proper modelling of the cooling cascade including molecular properties (anharmonicity, rotation...) is 
mandatory for band prediction and analysis. In this regard, there is still a lot of work to do in the laboratory and this not an easy task for either the experiment or the theoretical calculations. From the experimental point of view, there is a need for far-IR spectroscopy of large PAH molecules, but at $T<300 \mathrm{~K}$ to resolve (some) of the anharmonic structure. The calculations of the matrix of anharmonic parameters with DFT are very heavy and "tricky". Futhermore, for high temperatures, alternatives have to be found such as molecular dynamics with an approximated DFT scheme (Porezag et al. 1995; see also Pauzat and Basire et al., elsewhere in this volume). A lot of effort still needs to be dedicated to this subject both from the observational and laboratory points of view.

\section{References}

Allamandola, L.J., Tielens, A.G.G.M., \& Barker, J.R., 1989, ApJS, 71, 733

Bauschlicher, C.W., Boersma, C., Ricca, A., Mattioda, A.L., et al., 2010, ApJS, 189, 341

Bergin, E.A., Phillips, T.G., Comito, C., et al., 2010, A\&A, 521, L20

Désert, F.-X., Boulanger, F., \& Puget, J.L., 1990, A\&A, 237, 215

Draine, B.T., \& Li, A., 2001, ApJ, 551, 807

Draine, B.T., \& Li, A., 2007, ApJ, 657, 810

de Graauw, T., Helmich, F.P., Phillips, T.G., et al., 2010, A\&A, 518, L4

Griffin, M.J., Abergel, A., Abreu, A., et al., 2010, A\&A, 518, L3

Habing, H.J., 1968, BAIN, 19, 421

Herbig, G.H., 1995, ARA\&A, 33, 19

Joblin, C., Boissel, P., Léger, A., D’Hendecourt, L., \& Défourneau, D., 1995, A\&A, 299, 835

Joblin, C., Toublanc, D., Boissel, P., \& Tielens, A.G.G.M., 2002, Mol. Phys., 100, 3595

Joblin, C., \& Mulas, G., 2009, in: Interstellar dust properties: from fundamental studies to astronomical models, ed. F. Boulanger, C. Joblin, A. Jones, \& S. Madden, EAS Pub. Series, 35, 133

Kurucz, R.L., 1991, BAAS, 23, 1047

Le Petit, F., Nehmé, C., Le Bourlot, J., \& Roueff, E., 2006, ApJS, 164, 506

Malloci, G., Joblin, C., \& Mulas, G., 2007, Chem. Phys., 332, 353

Mattioda, A.L., Ricca, A., Tucker, J., Bauschlicher, C.W., \& Allamandola, L.J., 2009, AJ, 137, 4054

Mulas, G., 1998, A\&A, 338, 243

Mulas, G., Malloci, G., Joblin, C., \& Toublanc, D., 2006a, A\&A, 460, 93

Mulas, G., Malloci, G., Joblin, C., \& Toublanc, D., 2006b, A\&A, 456, 161

Pech, C., Joblin, C., \& Boissel, P., 2002, A\&A, 388, 639

Pilbratt, G.L., Riedinger, J.R., Passvogel, T., et al., 2010, A\&A, 518, L1

Pirali, O., Van-Oanh, N.-T., Parneix, P., Vervloet, M., \& Bréchignac, P., 2006, Phys. Chem. Chem. Phys., 8, 3707

Pirali, O., Vervloet, M., Mulas, G., Malloci, G., \& Joblin, C., 2009, Phys. Chem. Chem. Phys., 11, 3443

Poglitsch, A., Waelkens, C., Geis, N., et al., 2010, A\&A, 518, L2 
Porezag, D., Frauenheim, T., Köhler, T., Seifert, G., \& Kaschner, R., 1995, Phys. Rev. B, 51, 12947

Rouan, D., Léger, A., Omont, A., \& Giard, M., 1992, A\&A, 253, 498

Salama, F., Galazutdinov, G.A., Krełowski, J., Allamandola, L.J., \& Musaev, F.A., 1999, ApJ, 526, 265

Ysard, N., \& Verstraete, L., 2010, A\&A, 509, A12

Zhang, J., Han, F., Pei, L., Kong, W., \& Li, A., 2010, ApJ, 715, 485 